\title{
Performance Analysis of Front Bumper under Frontal Low Speed Impact
}

\author{
Ying Wang \\ School of Mechanical and Automotive Engineering, Shanghai University of Engineering Science, Shanghai, China \\ Email: wangying@sues.edu.cn
}

How to cite this paper: Wang, Y. (2021) Performance Analysis of Front Bumper under Frontal Low Speed Impact. Open Access Library Journal, 8: e6982.

https://doi.org/10.4236/oalib.1106982

Received: November 21, 2020

Accepted: January 1, 2021

Published: January 4, 2021

Copyright $\odot 2021$ by author(s) and Open Access Library Inc.

This work is licensed under the Creative Commons Attribution International License (CC BY 4.0).

http://creativecommons.org/licenses/by/4.0/ (c) (i) Open Access

\begin{abstract}
In this paper, the front bumper of a certain model was simulated and analyzed in low-speed frontal collision. HyperMesh software was used as the pre-processor, LS-DYNA was submitted for simulation calculation, and HyperView was used as the post-processor to view the output results, so as to analyze the performance of the automobile front bumper in low-speed frontal collision. At the same time, the influence of different beam thickness on collision performance is compared under the same condition, which provides an effective reference for subsequent bumper optimization and design.
\end{abstract}

\section{Subject Areas}

Automobile Engineering

\section{Keywords}

Car Front Bumper, Low-Speed Frontal Collision, Performance Analysis, Finite Element Method (FEM)

\section{1. 引言}

二十一世纪以来, 随着中国经济的高速发展, 汽车产业也迎来了飞速发 展。从 2009 年 2019 年, 中国汽车产销量已经连续十一年位居世界第一。随 之而来, 中国的汽车保有量也在不断攀升, 近十年中国民用汽车市场保有量 对比变化情况如图 1 所示。根据国家统计局发布的全国汽车市场保有量调查 统计数据显示, 截至 2019 年底, 中国民用汽车保有量累计达到 26,150 万辆, 而 2010 年仅为 7801.83 万辆 [1]。然而在我国汽车产业取得迅猛发展的同时, 所带来的安全隐患和环境污染问题也越发引人深思, 尤其是汽车引发的交通 事故已经逐渐成为严重危害公共安全的一个重要因素。据调查结果显示, 在 碰撞发生过程中平均发生碰撞概率最大的碰撞类型是正面碰撞, 其发生概率 
约占所有碰撞类型比例的一半[2]。汽车安全问题已成为世界各国政府和社会 普遍关注的重要问题, 因此加强汽车安全研究, 降低汽车碰撞事故伤害具有 重要的意义。随着汽车保有量的逐年攀升造成了城市的拥堵问题, 低速碰撞 出现的概率也相应提高, 而低速碰撞过程中首先发生接触的就是汽车保险杜。 随着汽车工业的发展, 对汽车保险杜力学性能的要求也随之增加, 对低速碰 撞中保险杜吸能特性和力学性能的研究意义重大。有限元法作为最有效的现 代复杂数值计算方法之一, 目前已广泛应用于大量的工程领域, 该计算方法 不仅具有很强的实际适用性, 还可以有效计算并分析各种具有复杂几何形状 和任意边界条件的结构, 同时还可以求解各种连续复杂物理场和多物理场的 耦合问题, 能够有效模拟各种工程试验方案, 在很大程度上节省了成本, 缩 短试验周期。因此本文采用有限元分析法, 对正面低速碰撞下汽车前保险杜 的性能进行分析。

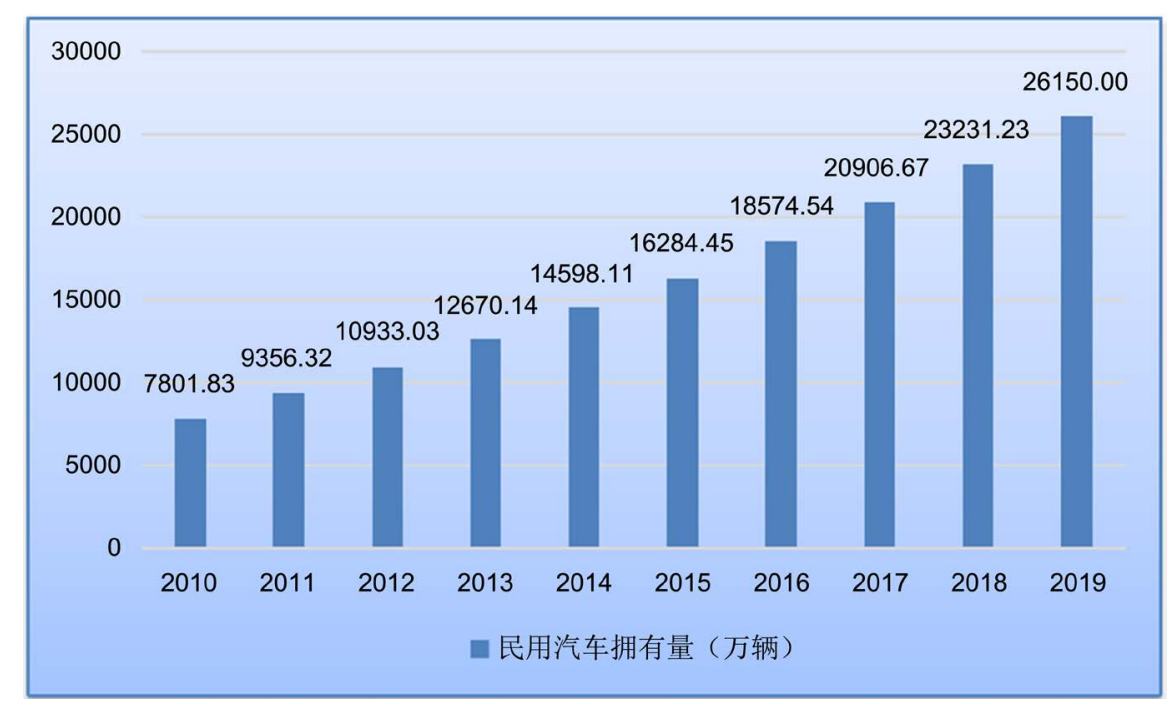

图 1. 近十年中国民用汽车保有量变化情况

\section{2. 有限元模型的构建}

\section{1. 前保险杠有限元模型}

本文以某款 SUV 为原车型, 运用 UG 建立了前保险杜几何模型, 如图 2 所示。模型包括保险杜横梁, 左右吸能盒。其中几何模型参数为: 保险杜 横梁长 $1105 \mathrm{~mm}$, 宽 $102 \mathrm{~mm}$, 两端厚度 $28 \mathrm{~mm}$, 由厚度为 $1.8 \mathrm{~mm}$ 的钢板焊 接而成; 吸能盒长 $130 \mathrm{~mm}$, 板材壁厚为 $2 \mathrm{~mm}$ 。模型文件保存为 STP 格式, 此格式是后处理软件的一种通用格式。另外, 有限元软件中没有固定的单位 制, 本文定义的各物理量单位制如表 1 所示。

\section{表 1. 各物理量单位制}

\begin{tabular}{cc}
\hline 物理量 & 单位制 \\
\hline 长度 & 毫米 $(\mathrm{mm})$ \\
时间 & 秒 $(\mathrm{s})$ \\
\hline
\end{tabular}




\section{Continued}

\begin{tabular}{cc}
\hline 质量 & 吨 $(\mathrm{t})$ \\
杨氏模量 & 兆帕 $(\mathrm{MPa})$ \\
密度 & 吨每立方毫米 $\left(\mathrm{t} / \mathrm{mm}^{3}\right)$ \\
\hline
\end{tabular}

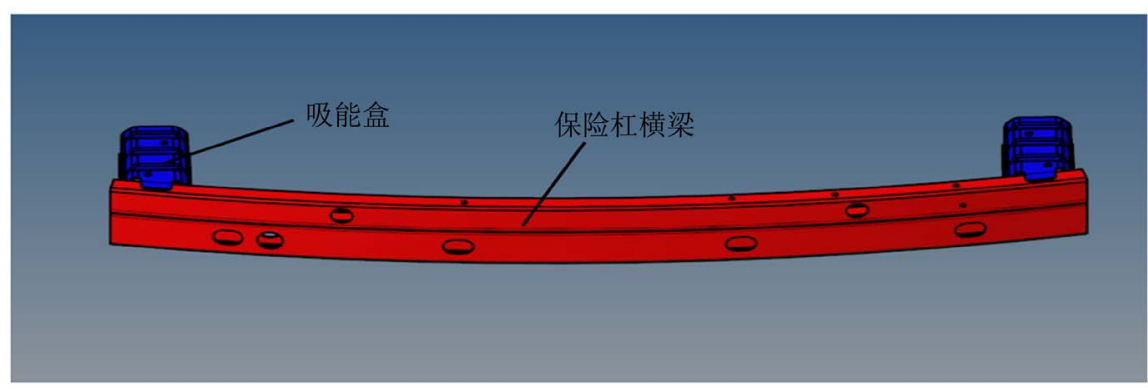

图 2. 汽车前保险杜几何模型

\section{2. 前保险杜有限元网格划分}

对模型进行几何清理后得到特征清晰、结构完整的中面模型, 以此为基 础, 采用四边形壳网格单元进行划分。在网格划分时, 尽量将三角形单元的 数量控制到最低, 以提高仿真分析计算的准确性, 因此网格单元尺寸最终确 定为 $3 \mathrm{~mm}$ 。为了使得划分的网格具有较高的质量, 采用手动的方式对得到的 较差的网格进行优化。本文得到的有限元网格模型的单元数为 45,900 , 节点 数为 46,505 , 部件质量因数 comp. QI 值为 0.01 。本文模型网格检查标准规范 如图 3 所示。

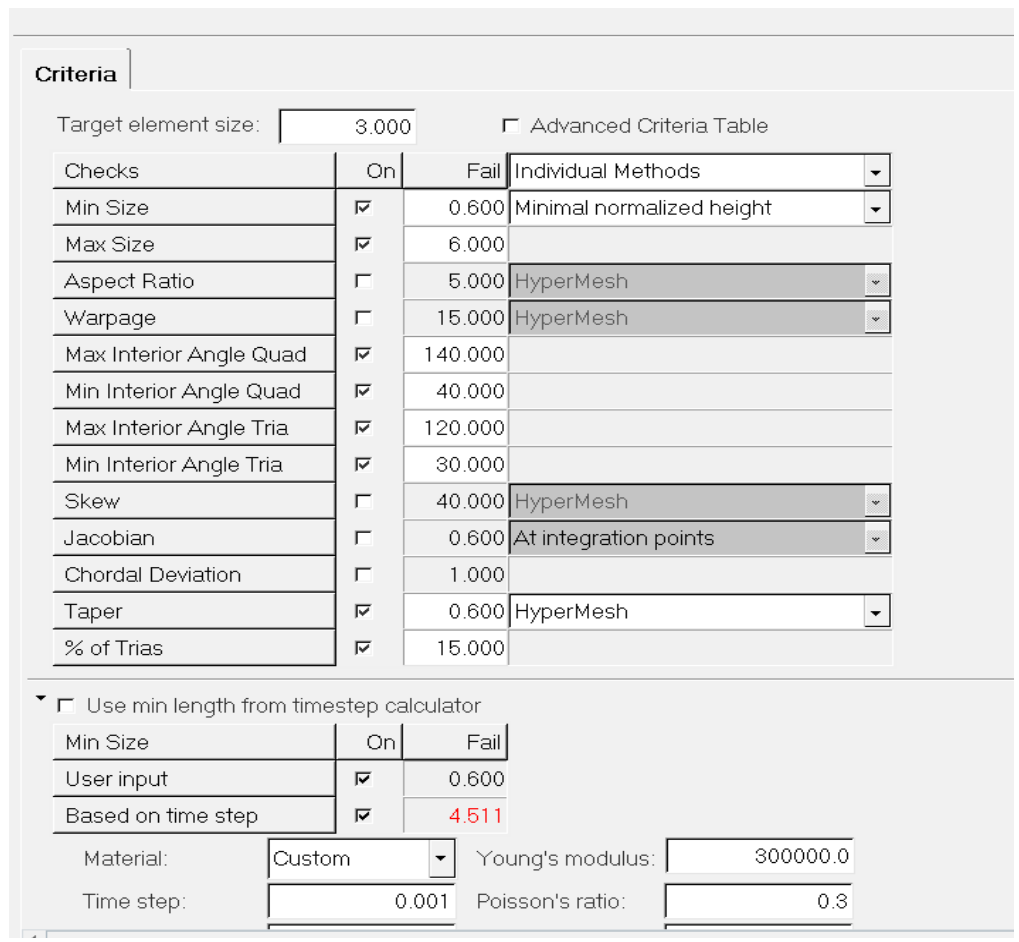

图 3. 网格检查标准设置 
为了更准确的模拟真实情况下汽车碰撞时的状态, 本文在吸能盒后方建 立了代表车身的刚体, 具体建模流程如下: 选择 HyperMesh 中 2D 面板下的 planes 工具建立刚性单元, 本模型中车体网格单元尺寸大小选择 $20 \mathrm{~mm}$, 命 名为 Car 组件, 并在这个组件下建立 mass 单元给模型加配重, 模拟实际情况 下整车的重量, Car 组件作为质量部件, 通过使用 ELEMENT MASS PART 工 具, 为模型赋予 $1.5 \mathrm{t}$ 的质量, 如图 4 所示。

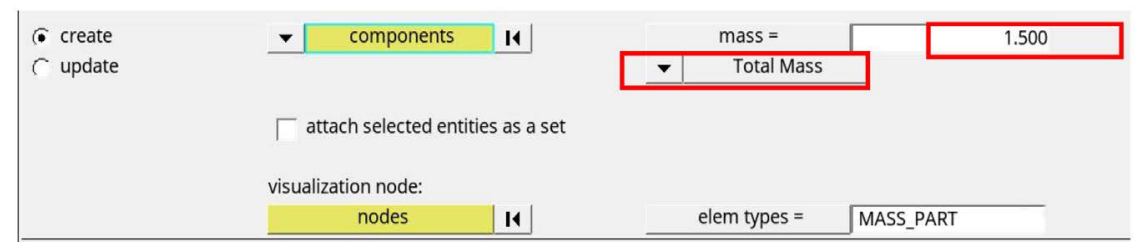

图 4. 模型质量赋予

\section{3. 材料属性定义}

为使模型仿真更加贴近实际, 本文借鉴了某主机厂根据国家试验标准进 行的拉伸和弯曲试验测试得到的材料应力 - 应变曲线, 将其导入到 HyperMesh 软件, 并显示在 Curves 部件中。模型具体材料如下: 保险杜横梁 以及吸能盒均为 24 号多线性弹塑性材料, 其是 LS-DYNA 材料库针对保险杜 模型的一种材料 [3], 后方代表车身的刚体采用 20 号刚体材料, 吸能盒材质为 HC340/590DP 冷轧双相钢, 是汽车轻量化中用量最多的材质之一, 是结构件、 加强件、防撞件的首选材料 [4]。保险杜横梁材质为 HC550/980DP 双相高强度 冷轧钢, 应力应变曲线如图 5 所示。HC340/590DP 材料在 Curves 中用四条不 同曲线表征在不同应变率下材料的应力应变的不同表现, 如图 6 所示, 其中, 横坐标为应变, 纵坐标为应力, 单位为 $\mathrm{MPa}$ 。模型各材料的参数如表 2 所示。

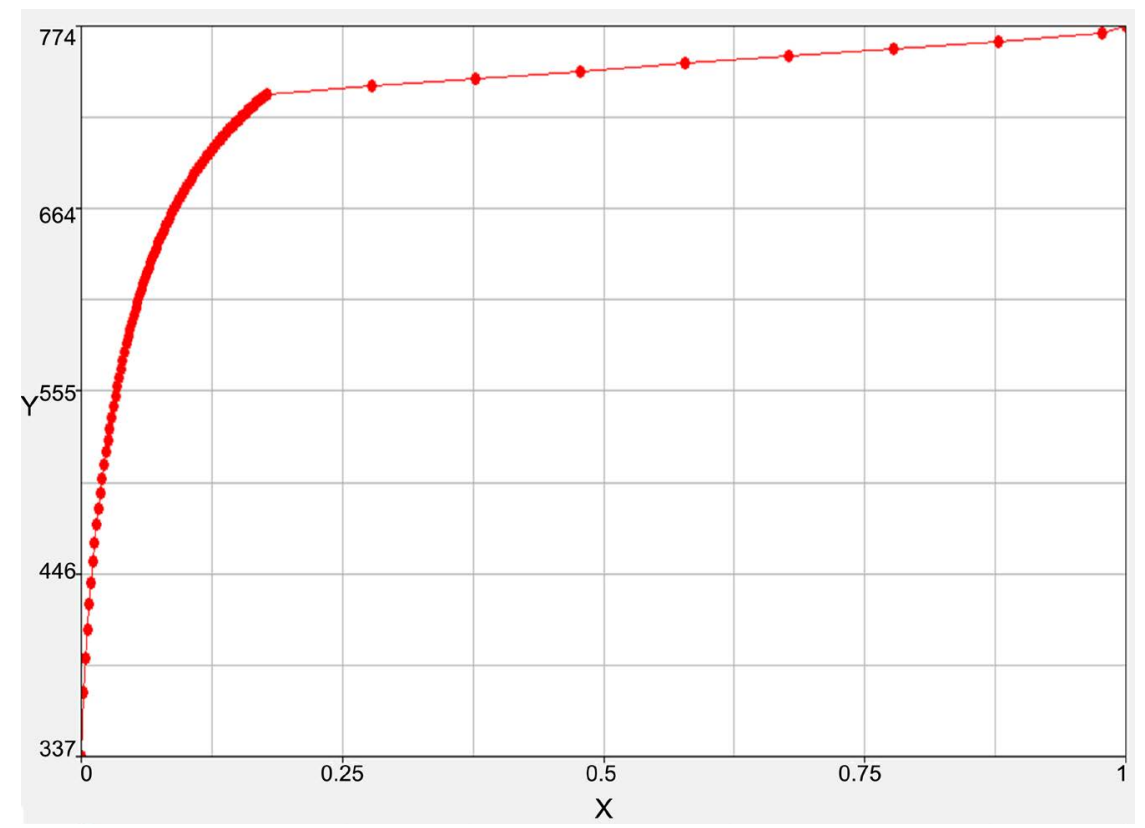

图 5. HC550/980DP 材料应力应变曲线(比例为 1:1) 


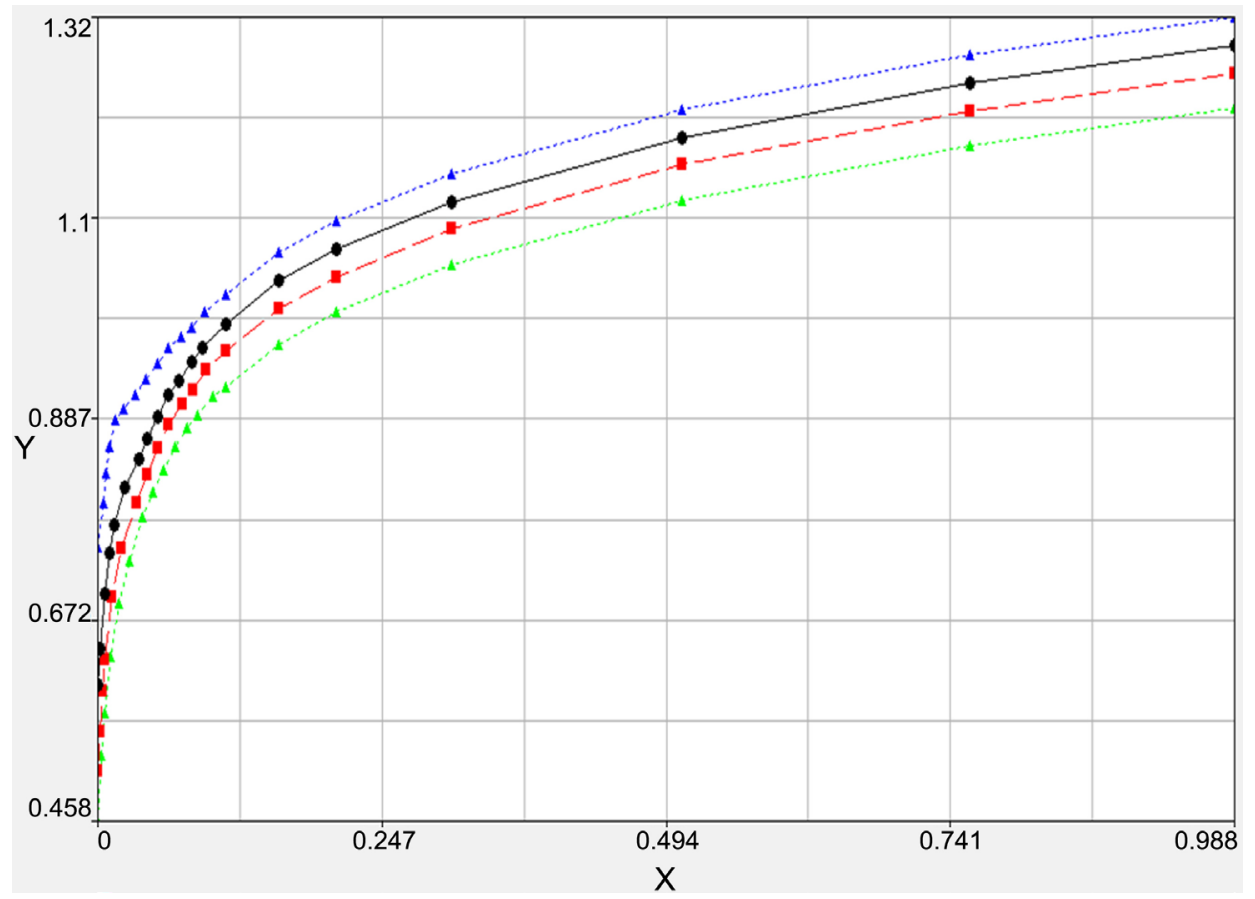

图 6. HC340/590DP 材料应力应变曲线( $\mathrm{Y}$ 方向缩放 1250 倍)

表 2. 各材料参数

\begin{tabular}{ccccc}
\hline & 材料 & $\begin{array}{c}\text { 密度 } \\
\rho\left(\mathrm{t} / \mathrm{mm}^{3}\right)\end{array}$ & $\begin{array}{c}\text { 弹性模量 } \\
\mathrm{E}(\mathrm{MPa})\end{array}$ & $\begin{array}{c}\text { 泊松比 } \\
\mathrm{u}\end{array}$ \\
\hline 保险杜横梁 & HC340/590DP & $7.850 \mathrm{e}-09$ & 205,000 & 0.3 \\
吸能盒 & HC550/980DP & $7.842 \mathrm{e}-09$ & 207,000 & 0.29 \\
刚体 & MATL20 & $7.800 \mathrm{e}-09$ & 210,000 & 0.3 \\
\hline
\end{tabular}

\section{4. 边界条件的设定}

本模型中, 在 HyperMesh 软件中定义边界条件, 定义的类型包括设置刚 性墙以及赋予初始速度。刚性墙的创建通过 Analysis 面板下的 rigid walls 功 能实现, 刚性墙设在有限元模型运动方向的正前方, 作用方向与汽车运动方 向相反, 且刚性墙设置为无限大, 并与所有组件进行接触设置。赋予初始速 度的作用是保证前保险杜模型能撞向刚性墙, 本文参考 ECE R42 法规, 设置 初始速度为 $4 \mathrm{~km} / \mathrm{h}$, 在 HyperMesh 中点击 Load Collectors 菜单, 选中所有点 的集合, 在 $X$ 方向设置初始速度为 $-1111 \mathrm{~mm} / \mathrm{s}$, 如图 7 所示。

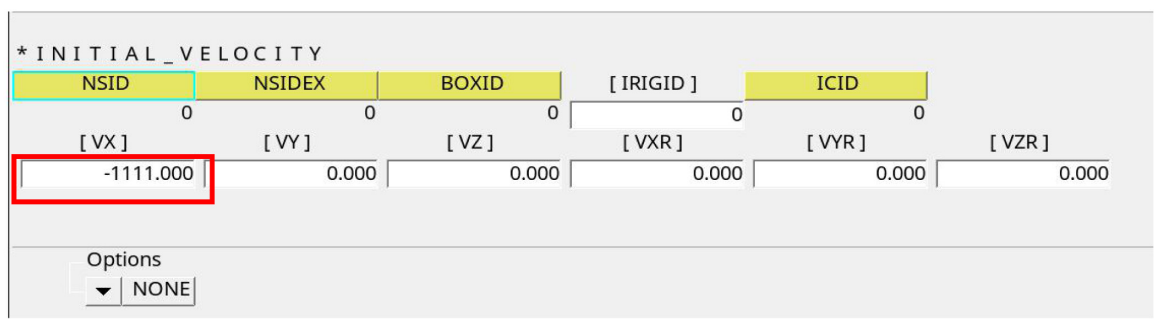

图 7. 初始速度设置 


\section{3. 有限元仿真分析}

仿真分析的研究方法是以现代计算机模拟技术、系统技术、相似原理、 以及仿真应用领域的有关技术作为基础, 利用模型对真实系统进行仿真研究 的一门多学科综合性技术 [5]。有限元仿真分析的过程就是通过建立有限元模 型来形成文件, 并提交给求解器计算。

\section{1. 碰撞变形分析}

在低速碰撞过程中, 前保险杜系统在 $0 \sim 0.1 \mathrm{~s}$ 内的变形过程如图 8 所示。

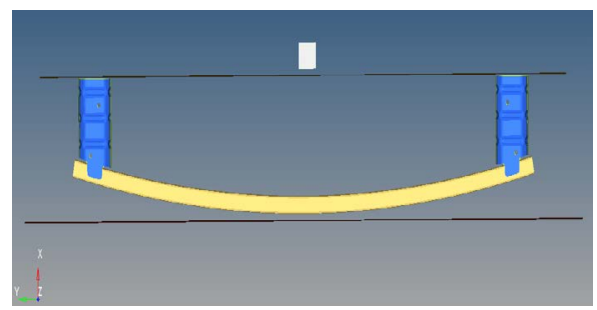

(a) $t=0 s$

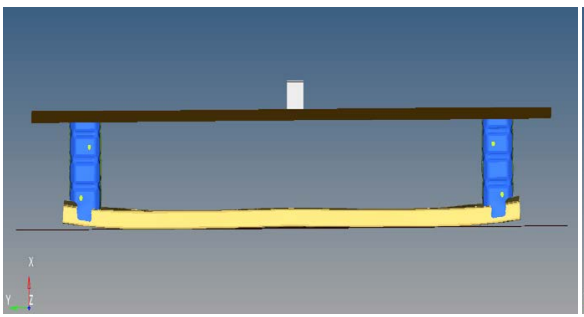

(c) $\mathrm{t}=0.08 \mathrm{~s}$

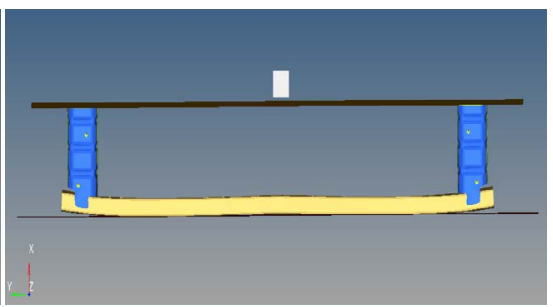

(b) $t=0.06 s$

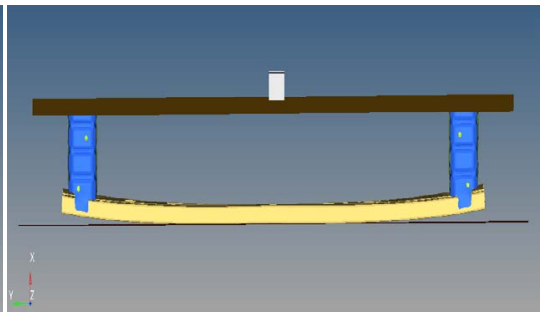

(d) $\mathrm{t}=0.1 \mathrm{~s}$

图 8. 碰撞过程中前保险杠的变形过程

在保险杜撞击刚性墙过程中, 保险杜横梁发生明显变形, 吸能盒几乎不 变形, 保险杜系统的碰撞安全性能较好。在 $0 \sim 0.06 \mathrm{~s}$ 之间保险杜横梁中间的 弧形突出部分首先与刚性墙接触, 刚性墙阻碍其运动, 横梁发生明显的弯曲 变形, 由弧形构造被压成平直形状, 随着横梁与刚性墙进一步的接触, 中间 弧形部分因受到挤压, 弯曲继续变大。在 0.06 0.1 s 之间, 横梁存在一个极小 的自动回弹修复的过程, 这与横梁的多线性弹塑性材料相关。由于碰撞速度 较低, 吸能盒还未发生明显变形碰撞过程就已经结束, 符合汽车发生正面碰 撞过程的变形规律。

\section{2. 碰撞能量分析}

本模型中前保险杜能量随时间变化曲线和能量数值分别如图 9 和表 3 所 示。在碰撞过程中前保险杜系统总能量保持不变, 沙漏能维持在 0 附近, $\mathrm{t}=0.1$ $\mathrm{s}$ 时, 沙漏能与总能量的比值为 $0.17 \%$, 小于 $5 \%$, 说明模型是准确有效的, 本次计算结果可靠。随着保险杜横梁与刚性墙接触, 系统动能逐渐减少, 内 能逐渐增加, 在 $0.068 \mathrm{~s}$ 时动能达到最小, 为 $13.832 \mathrm{~J}$, 内能达到最大, 为 922.631 $\mathrm{J}$, 之后保险杜横梁由于弹性作用慢慢恢复原状, 动能开始增加, 内能逐渐减 少, 碰撞过程结束, 仿真结果与实际相符。 


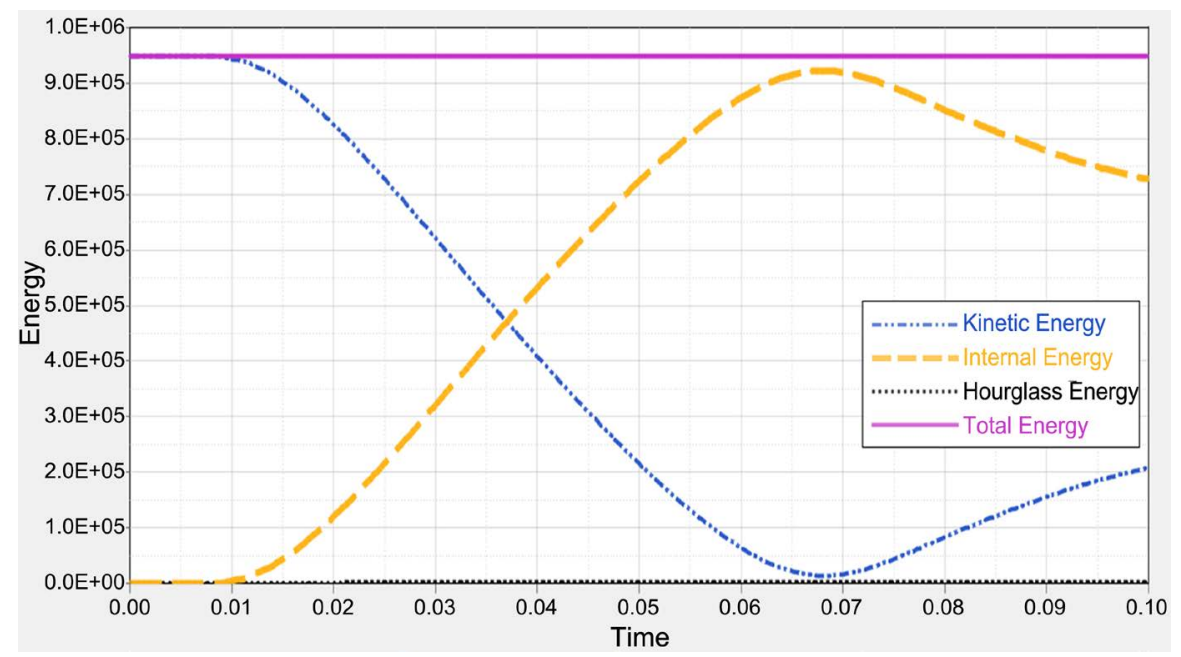

图 9. 前保险杠能量随时间变化曲线

表 3. 能量数值

\begin{tabular}{ccc}
\hline 能量类型 & $\mathrm{t}=0 \mathrm{~s}$ & $\mathrm{t}=0.1 \mathrm{~s}$ \\
\hline 动能 $/ \mathrm{J}$ & 948,187 & 206,866 \\
内能/J & 0 & 727,317 \\
总能量 $/ \mathrm{J}$ & 948,187 & 948,130 \\
沙漏能/J & 0 & 1626.03 \\
\hline
\end{tabular}

前保险杜横梁及吸能盒内能随时间变化曲线如图 10 所示。 $\mathrm{t}=0.068 \mathrm{~s}$ 时, 横梁内能达到峰值为 $677.230 \mathrm{~J}$, 吸能盒内能为 $245.464 \mathrm{~J}$, 此时, 保险杜横梁 和吸能盒的内能占系统总内能百分比分别为 $73.40 \%$ 和 $26.60 \%$ 。这表明在 4 $\mathrm{km} / \mathrm{h}$ 的低速碰撞过程中, 横梁将大部分的动能转变为了自身的变形能, 横梁 是前保险杜组成部件中的主要吸能构件, 该工况下吸能盒的吸能作用还未充 分发挥, 该保险杜的碰撞性能较好。

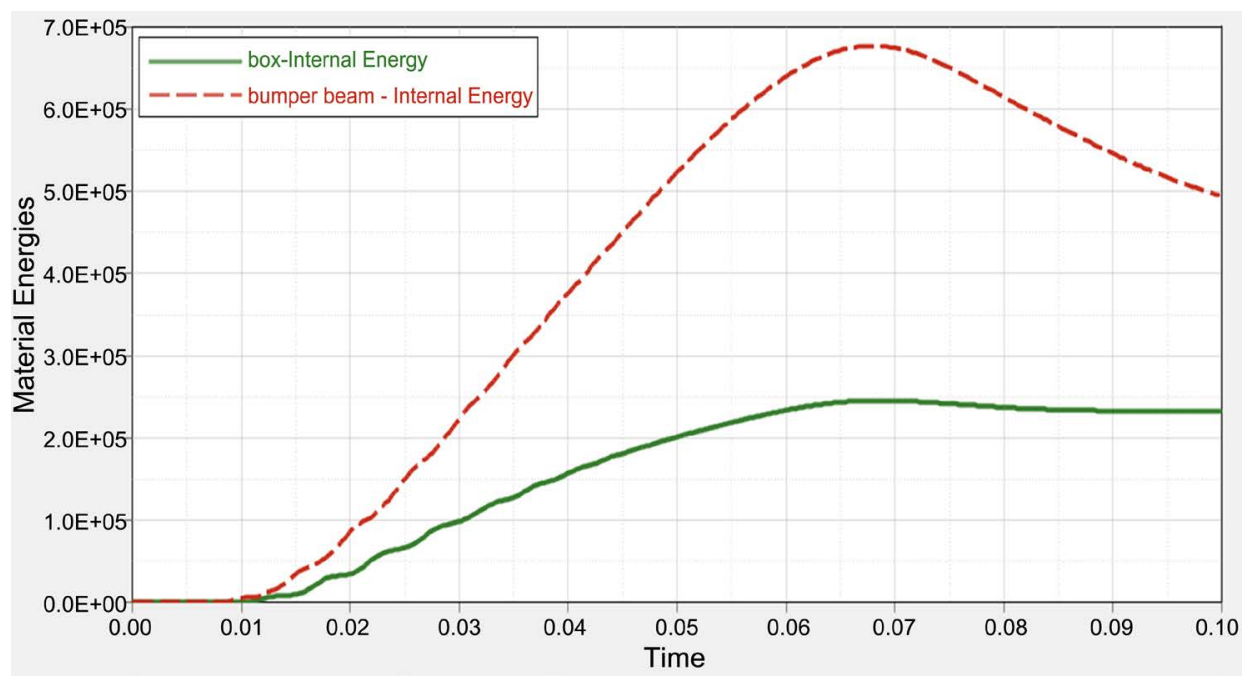

图 10. 前保险杜内能随时间变化曲线 


\section{3. 碰撞位移分析}

汽车前保险杜与刚性墙碰撞过程中, 保险杜位移云图以及车体位移、速 度随时间变化曲线分别如图 11、图 12、图 13 所示。

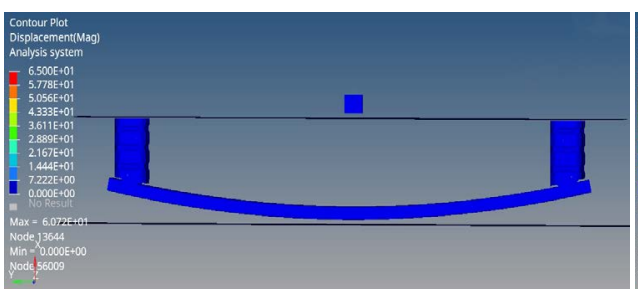

(a) $\mathrm{t}=0 \mathrm{~s}$

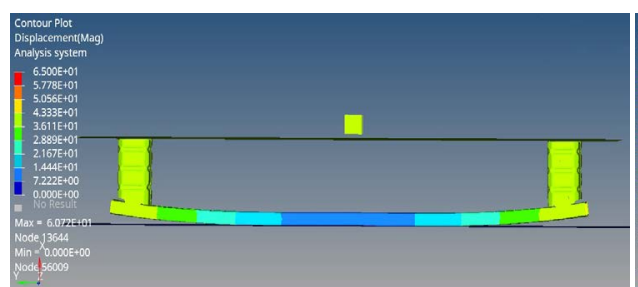

(c) $\mathrm{t}=0.04 \mathrm{~s}$

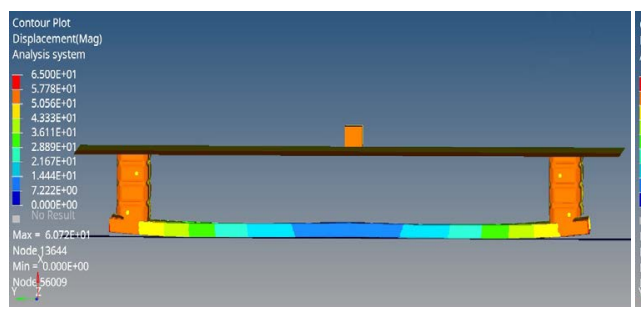

(e) $\mathrm{t}=0.08 \mathrm{~s}$

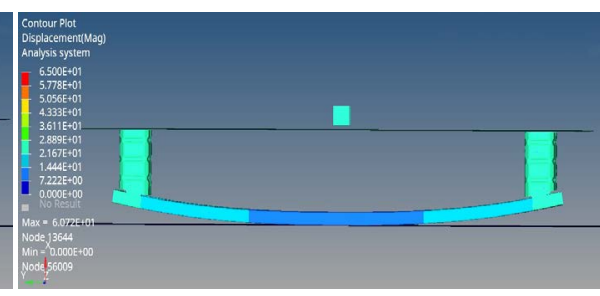

(b) $\mathrm{t}=0.02 \mathrm{~s}$

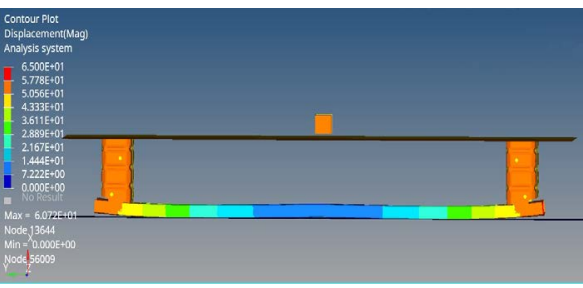

(d) $\mathrm{t}=0.06 \mathrm{~s}$

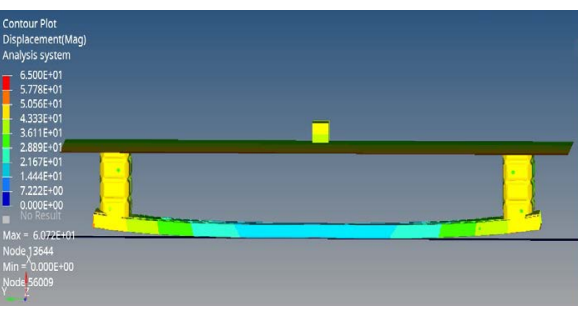

(f) $\mathrm{t}=0.1 \mathrm{~s}$

图 11. 前保险杜位移云图

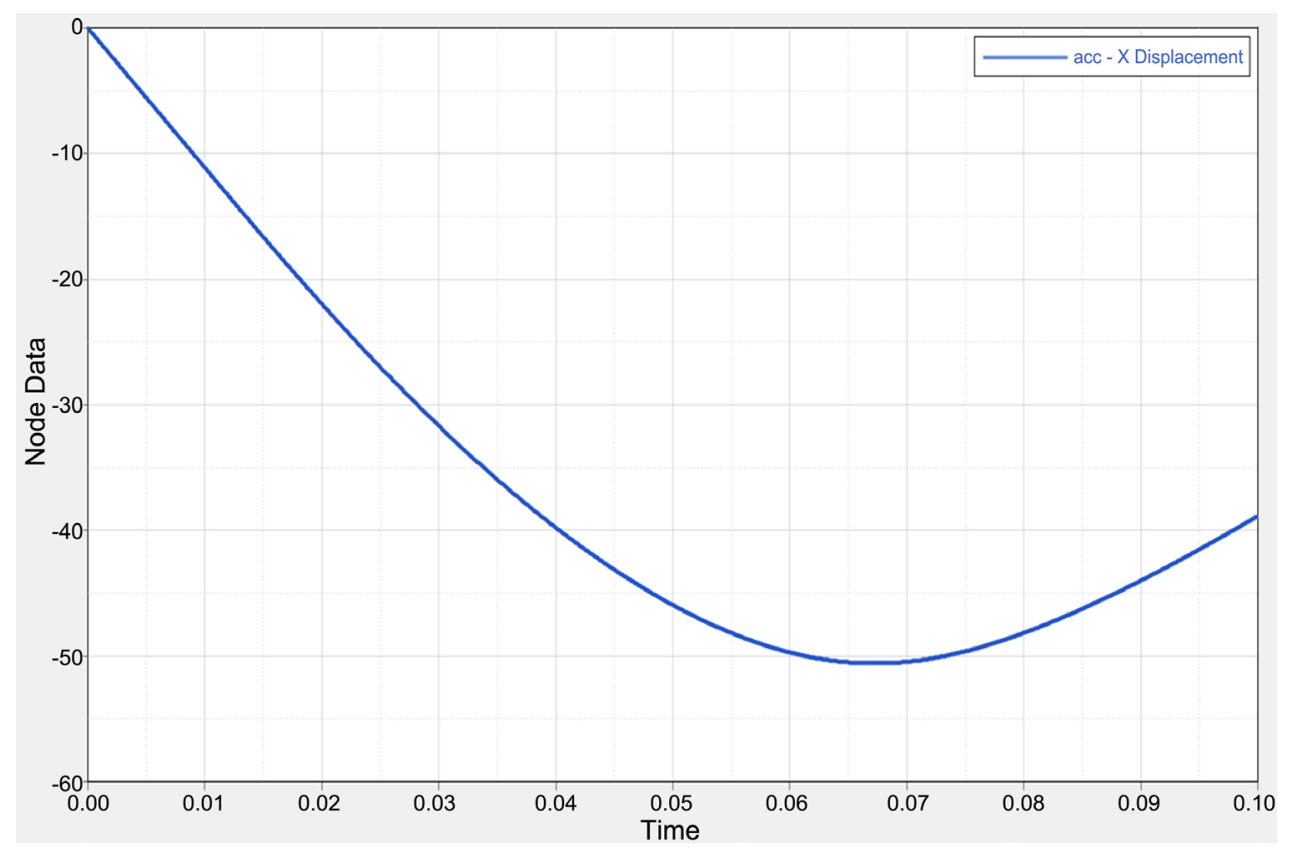

图 12. 车体位移随时间变化曲线 


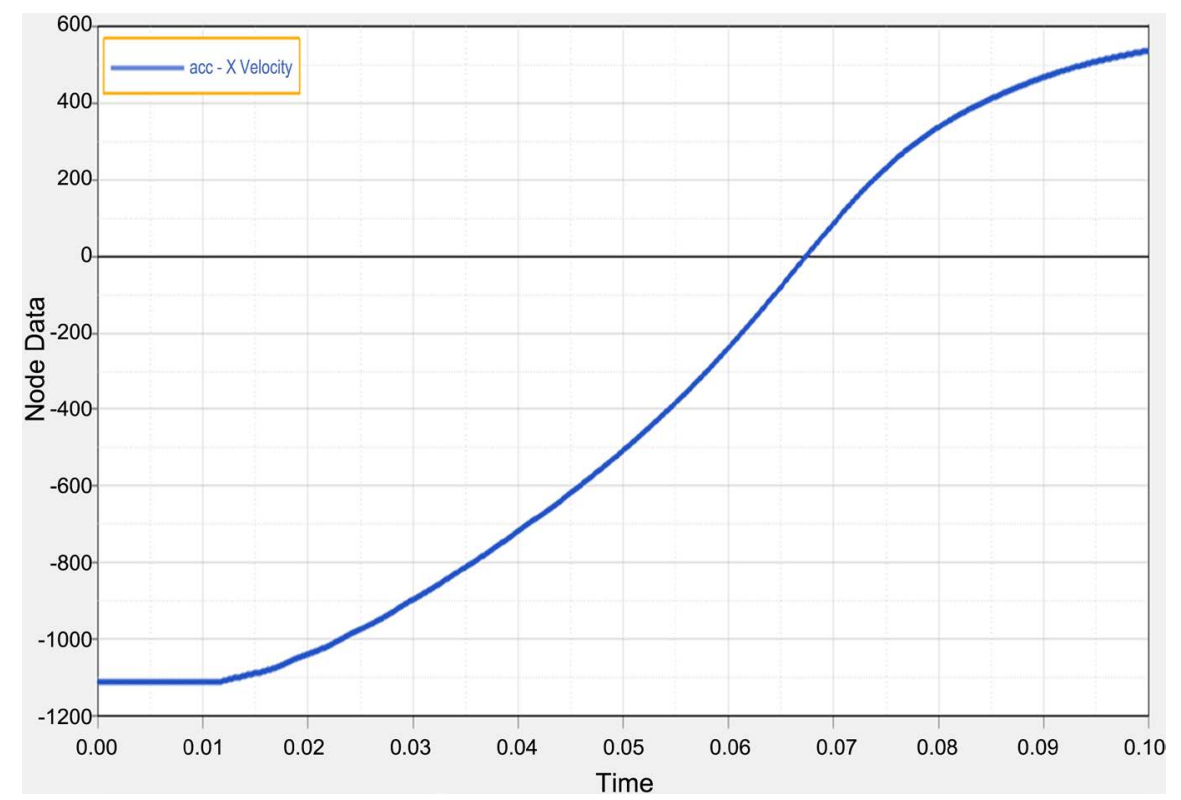

图 13. 车体速度随时间变化曲线

由图 12 可知, 系统碰墥过程较稳定, 车体位移先随时间增大至最大值 $50.591 \mathrm{~mm}$, 此时对应的前保险杜(横梁及吸能盒)压缩量最大, 且此时保险杜 具有一个微小的反弹速度, 之后横梁开始与刚性墙分离, 保险杜内能转化为 动能, 位移开始减小。说明前保险杜后期产生了弹性变形, 与保险杜系统的 位移云图显示结果一致。

\section{4. 碰撞加速度分析}

速度在单位时间内变化的大小会引起加速度变化的快慢, 加速度值越大, 冲击能力越强, 对乘员的伤害就越大, 有效控制碰撞加速度大小是汽车安全 所考虑的问题[6]。位移的求导为速度, 本文在 HperView 中采用对位移曲线 进行二次求导、滤波的方式得到加速度曲线, 单位为 $g$ ( $\mathrm{g}$ 为重力加速度), 如 图 14 所示。

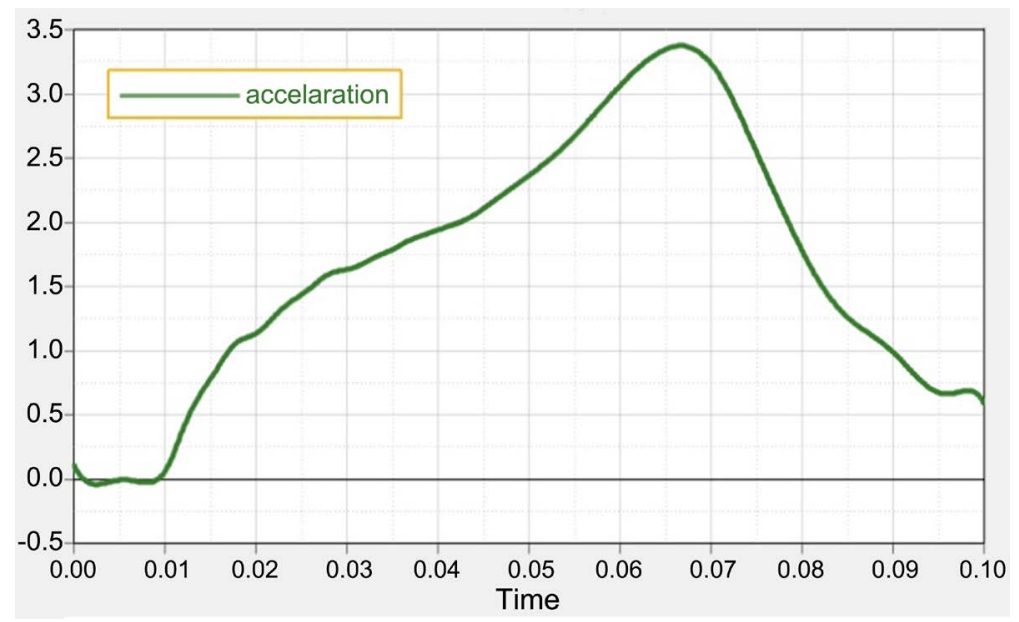

图 14. 加速度随时间变化曲线 
仿真计算得出的加速度大小的峰值为 $3.37 \mathrm{~g}$, 约出现在 $0.067 \mathrm{~s}$ 处, 持续 时间很短。汽车碰撞过程中加速度值越大, 乘员越容易在碰撞过程中受伤。 一般而言, 加速度曲线峰值越低, 持续时间越短, 曲线越光滑, 则该汽车对 应的正碰结构耐撞性越好[7]。

\section{4. 横梁厚度对碰撞性能的影响分析}

保险杜横梁一般用作低速碰撞下的安全保护系统, 其良好的结构能在很 大程度上提高车辆的被动安全性, 保护车辆与乘客安全。在保险杜碰撞安全 性研究中, 材料强度、厚度的合理匹配通常作为首要考虑的因素, 为了进一 步研究前保险杜系统的碰撞性能, 本文通过改变横梁壁厚探讨保险杜横梁厚 度对碰撞性能的影响。

\section{1. 碰撞内能比较}

前文分析中保险杜横梁的厚度为 $1.8 \mathrm{~mm}$, 在其它条件不变的情况下, 分 别取横梁厚度为 $2 \mathrm{~mm}$ 和 $2.3 \mathrm{~mm}$ 与原始厚度进行对比仿真分析, 得出不同横 梁厚度下的前保险杜系统内能曲线及内能数值对比, 如图 15、表 4 所示。

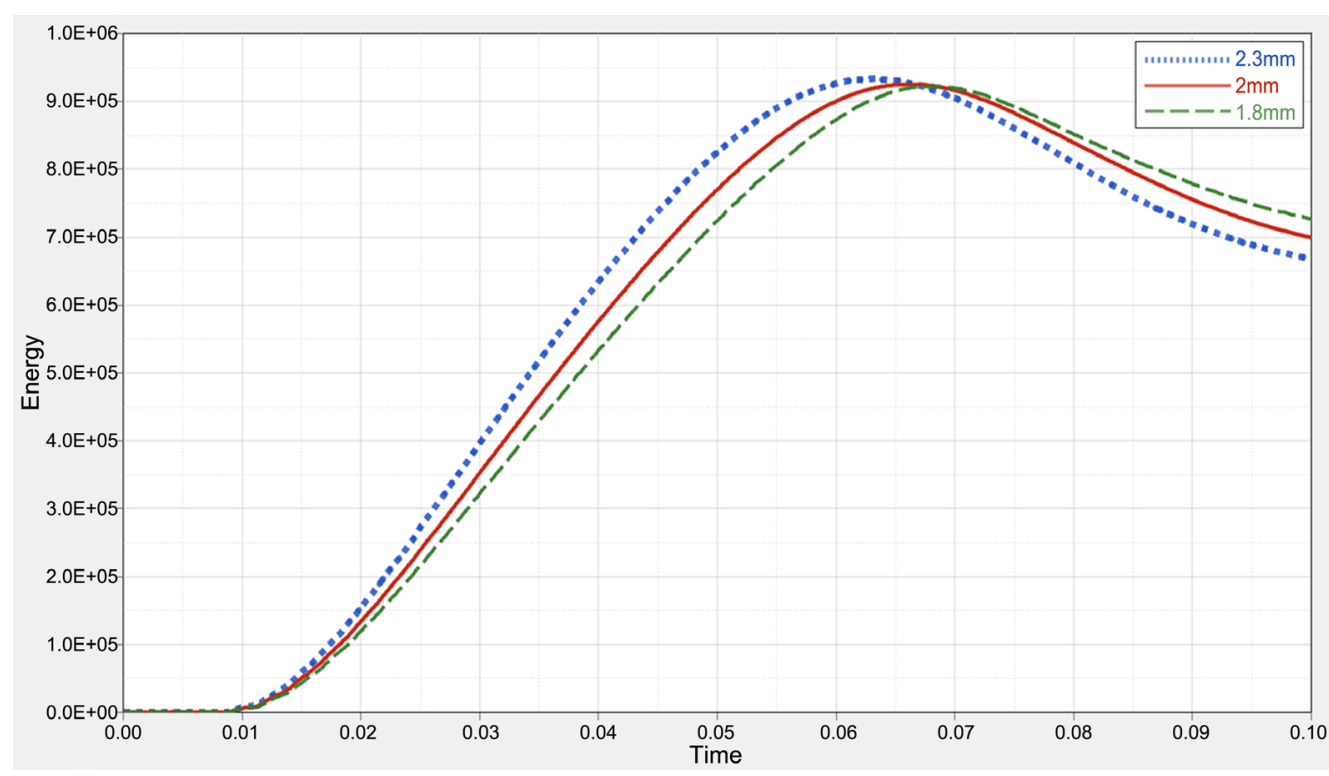

图 15. 不同横梁厚度下的前保险杜系统内能曲线

表 4. 最大内能及所需时间对比

\begin{tabular}{ccc}
\hline 横梁厚度 $/ \mathrm{mm}$ & 内能最大值 $/ \mathrm{J}$ & 内能达到最大值所需时间 $/ \mathrm{s}$ \\
\hline $\mathrm{T}=1.8$ & 922,631 & 0.068 \\
$\mathrm{~T}=2$ & 925,508 & 0.066 \\
$\mathrm{~T}=2.3$ & 932,889 & 0.063 \\
\hline
\end{tabular}

由图 15 可知, 横梁厚度为 $1.8 \mathrm{~mm} 、 2 \mathrm{~mm}$ 和 $2.3 \mathrm{~mm}$ 的前保险杜系统的 内能曲线的总体趋势大致相同, 横梁厚度为 $2 \mathrm{~mm}$ 和 $2.3 \mathrm{~mm}$ 的保险杜的内能 
峰值比厚度为 $1.8 \mathrm{~mm}$ 的保险杜内能峰值大。该结果表明随着横梁厚度的增 加, 保险杜系统吸收的能量越多。然而横梁厚度并非越大越好, 如表 4 所示, 横梁厚度为 $1.8 \mathrm{~mm}$ 与厚度为 $2 \mathrm{~mm}$ 的内能相差不大, 且达到内能最大值时间 几乎相等, 而厚度的增加在一定程度上会使车重增加, 不符合汽车环保和经 济的理念。

\section{2. 碰撞变形比较}

图 16 所示为不同横梁厚度的保险杜系统在内能曲线最高点时的变形图。 由图可知, 横梁厚度为 $1.8 \mathrm{~mm} 、 2 \mathrm{~mm}$ 和 $2.3 \mathrm{~mm}$ 的三种情况下保险杜的变形 只存在细微的差别。

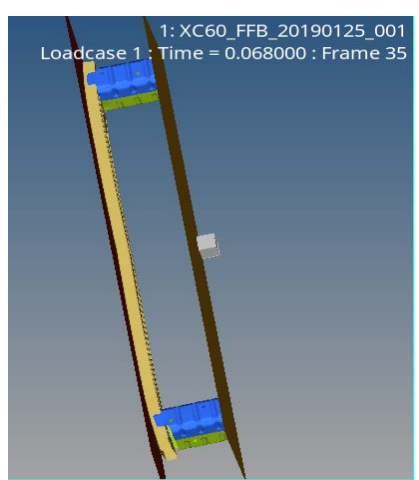

(a) 横梁厚 $1.8 \mathrm{~m}$

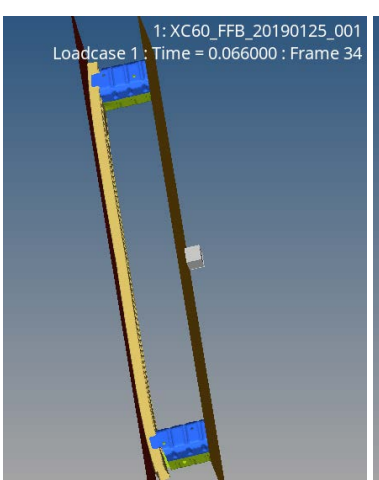

(b) 横梁厚 $2 \mathrm{~mm}$

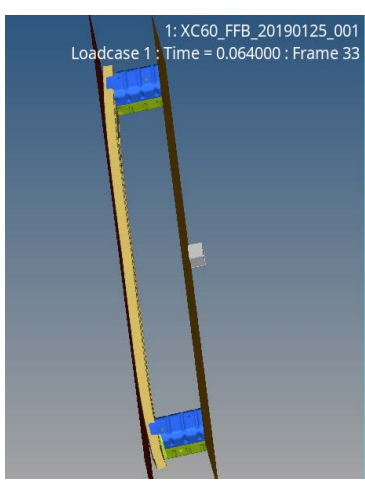

(c) 横梁厚 $2.3 \mathrm{~mm}$

图 16. 不同横梁厚度下的前保险杜系统变形图

图 17 为不同横梁厚度下吸能盒的内能曲线图, 由图可知, 横梁厚度增加 时, 吸能盒吸收的能量也随之增加, 出现这种情况的原因是: 横梁厚度的增 加使横梁刚度也随之增加, 当碰撞发生时, 刚度越大的横梁变形越小, 吸收

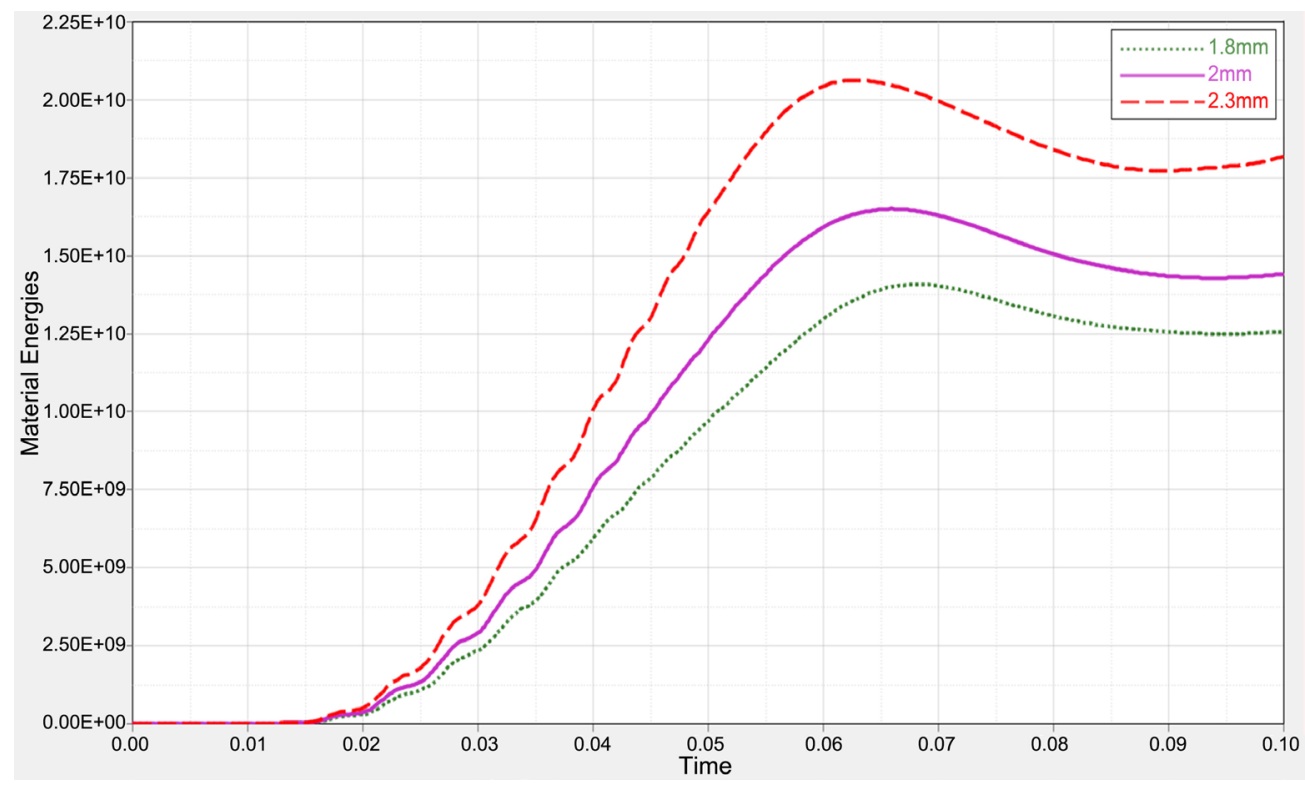

图 17. 吸能盒内能曲线图 
碰撞过程中的能量便越少, 横梁作为承载件将残余的冲击力传递到吸能盒上, 吸能盒在力的作用下发生变形, 吸收部分能量。在汽车设计的安全性能研究 理论中, 保险杜系统在碰撞过程中吸能盒的变形越大, 可能会导致后端部件 发生碰撞, 不能很好的保护车体。

\section{5. 结论}

随着汽车保有量的持续增长, 汽车道路交通事故成为了一个饱受关注的 问题, 以至于人们对汽车被动安全性的要求越来越高。据调查显示, 汽车正 面碰撞是所有碰撞类型中发生最频繁的类型之一 $[8]$ 。汽车前保险杜作为汽车 正面碰撞的重要吸能装置, 对保护乘员安全起到至关重要的作用, 其吸能效 果也是评价汽车安全性能的重要参数之一。

在正面低速碰撞仿真中，保险杜横梁通过产生弯曲变形，吸收碰撞中很 大一部分能量, 保险杜横梁起主要吸能作用。保险杜横梁厚度并不是越大越 好, 保险杜的碰撞安全性能与横梁的厚度不成正比关系。横梁厚度需与整车 匹配，才能实现最理想的保护效果。

\section{Conflicts of Interest}

The author declares no conflicts of interest regarding the publication of this paper.

\section{References}

[1] 中华人民共和国国家统计局[EB/OL]. http://www.stats.gov.cn/

[2] 杨辉. 汽车碰撞试验法规综述 [J]. 上海标准化, 2006(6): 16-20.

[3] Vasdravellis, G., Karavasilis, T.L. and Uy, B. (2014) Design Rules, Experimental Evaluation, and Fracture Models for High-Strength and Stainless-Steel Hourglass Shape Energy Dissipation Devices. Journal of Structural Engineering, 140, 733-741. https://doi.org/10.1061/(ASCE)ST.1943-541X.0001014

[4] 郑宏良, 潘海涛, 张辉. HC340/590DP 钢板激光焊可行性分析 [J]. 电焊机, 2017, 47(11): 125-127.

[5] 杨永生. 汽车保险杜系统低速碰撞性能研究[D]: [硕士学位论文]. 哈尔滨: 哈尔 滨工程大学, 2009.

[6] 刘成阔. 汽车保险杜系统碰撞分析及优化设计[D]: [硕士学位论文]. 大连: 大连 交通大学, 2017.

[7] 杨成国. 某车型的正面碰撞模拟仿真分析[D]: [硕士学位论文]. 沈阳: 沈阳工业 大学, 2017.

[8] 中国汽车技术研究中心. 这个汽车安全发展报告(2014) [M]. 北京: 社会科学文 献出版社, 2014: 32-34. 